\title{
Genome announcement: complete genome sequence of a novel Mupapillomavirus, HPV204
}

\author{
Boštjan J. Kocjan ${ }^{1}$, Anja Šterbenc ${ }^{1}$, Lea Hošnjak ${ }^{1}$, Diego Chouhy², Elisa Bolatti², Adriana A. Giri², Mario Poljak ${ }^{1 凶}$
}

\begin{abstract}
Human papillomaviruses (HPVs) are small, non-enveloped viruses with a circular double-stranded DNA genome, etiologically associated with various benign and malignant neoplasms of the skin and mucosa. As of May 30, 2015, 201 different HPV types had been completely sequenced and officially recognized and divided into five PV-genera: Alpha-, Beta-, Gamma-, Mu-, and Nupapillomavirus. The Mupapillomavirus genus currently consists of only two HPV types: HPV1 and HPV63, identified in 1980 and 1993 , respectively, both associated with sporadic cases of cutaneous warts. In this preliminary study, we announce the complete genome sequence of a novel HPV type, now officially recognized as HPV204. Based on preliminary data, the genome of HPV204 comprises a total of 7,227 bp and contains five early open reading frames (E1, E2, E4, E6, and E7) and two late ORFs (L1 and L2). No E5 ORF could be identified. Preliminary HPV204 clusters to the Mu-PV genus, species Mu-3.
\end{abstract}

Keywords: human papillomavirus, HPV, HPV204, Mupapillomavirus, genomic characterization

Received: 10 April 2015 | Returned for modification: 26 April 2015 | Accepted: 12 May 2015

Human papillomaviruses (HPVs) are a large family of small, genetically diverse DNA viruses that can cause benign and malignant proliferations of the skin and mucosal epithelia (1, 2). HPVs are taxonomically classified into genera, species, and types based on sequence similarities of the highly conserved L1 genomic region. A novel HPV type is recognized as such when its genome has been fully cloned and deposited to the International HPV Reference Center (www.hpvcenter.se) and its L1 nucleotide (nt) sequence shows less than 90\% identity to any other known HPV type (1, 3). As of May 30, 2015, 201 different HPV types belonging to 49 viral species had been completely sequenced and officially recognized and divided into five papillomaviruses (PV)-genera: Alphapapillomavirus (Alpha-PV), Betapapillomavirus (Beta-PV), Gammapapillomavirus (Gamma-PV), Mupapillomavirus (Mu-PV), and Nupapillomavirus (Nu-PV) (4). Several additional HPVs have been completely sequenced, mainly using next-generation sequencing, but are not yet officially recognized (5). These are listed in the PapillomaVirus Episteme (PaVE) database, together with the sequences of animal PV genomes (5).

The $M u$-PV genus currently consists of only two cutaneotrophic HPV types: HPV1 (species Mu-1) and HPV63 (species Mu-2), which are etiologically associated with sporadic cases of skin warts (6). HPV1 was identified in 1980 in tissue specimens of deep plantar warts (7) and fully sequenced 2 years later (8). HPV63 was identified and fully sequenced in 1993 from a tissue specimen of a keratotic lesion (9). In recent years, the number of Gamma-PVs has been growing most rapidly, with 81 completely sequenced types to date, followed by Alpha- and Beta-PV genera, which consist of 65 and 51 recognized types, respectively $(4,10)$. In contrast, only a single putatively novel $\mathrm{Mu}$-PV sequence has been identified in recent years (11), although several modern molecular approaches for virus detection, including metagenomic sequencing, were used (4). We report here the preliminary genome sequence of a novel $\mathrm{Mu}$-PV type HPV2O4.

A partial 200-bp L1 gene nt sequence (ENA accession number: FJ947082) similar to both Mu-PVs (HPV1 and HPV63) was origi- nally identified in 2009 using FAP primers and single tube "hanging droplet” PCR (11). A complete viral genome was amplified in 2014 using inverse long-range PCR with primer pair FJ947082LR-F (5'-GGTATGGGCATTAAGAGGTTTA-3', nt 5,484-5,505) and FJ947082-LR-R (5'-AAGCGTTCTGTTTCAGGATTAA-3'， nt 5,4835,462) and the Platinum Taq DNA Polymerase High Fidelity Kit (Invitrogen, Carlsbad, CA). The resulting amplicon of approximately $8 \mathrm{kbp}$ was gel-purified, cloned into a plasmid vector using TOPO XL PCR Cloning Kit (Invitrogen), and sequenced on both strands at Microsynth AG (Balgach, Switzerland) using the primer-walking strategy. The nt sequence of the complete viral genome was assembled using Vector NTI Advance 11 software package (Invitrogen). PV-specific open reading frames (ORFs) were determined using the ORF Finder Tool (http://www.ncbi.nlm.nih.gov/gorf/ gorf.html). Preliminary phylogenetic relationships were inferred by Bayesian analysis, based on the entire L1 gene nt sequence of HPV2O4 and 61 officially recognized HPV types from the Alpha-, Beta-, Gamma-, $\mathrm{Mu}$-, and $\mathrm{Nu}$-PV genera. Markov Chain Monte Carlo simulations were performed on 107 generations, sampling one state every 1,000 generations, with a burn-in of $10 \%$. The evolutionary substitution model was set as rtREV $+\Gamma+\mathrm{I}$.

A reference clone, containing the complete genome of HPV204, was deposited in the HPV Reference Center at the Karolinska Institute in Sweden in October 2014, where its nucleotide sequence was confirmed and the type officially designated in January 2015 (http://www.hpvcenter.se/html/refclones.html).

Based on preliminary data, the genome of HPV204 comprises a total of 7,227 bp and contains five early ORFs (E1, E2, E4, E6, and $\mathrm{E}_{7}$ ), coding proteins involved in viral replication, transcription, and transformation, and two late ORFs (L1 and L2), coding proteins of the capsid. No E5 ORF that is present in some PVs (12) could be identified. The non-coding long control region (LCR) that contains elements responsible for controlling transcription and replication of the viral genome was identified between the L1 and E6 ORFs. Preliminarily, with an L1 nt sequence identity of $66.3 \%$ and $66.7 \%$ to HPV1 and HPV63, respectively, HPV2O4 is 
taxonomically classified as a novel $M u$-PV type in a new species $M u-3$. As shown in Figure 1, our preliminary phylogenetic analysis of HPV204 and 61 HPV L1 nt sequences revealed that HPV2O4 clusters with HPV1 and HPV63, suggesting that the novel virus is most probably an indisputable member of the $\mathrm{Mu}$-PV genus.

In conclusion, the identification of HPV2O4 expands the current knowledge of the genetic diversity of the $\mathrm{Mu}$-PV genus and provides further information for the development of molecular tools for identifying and isolating novel $M u$-PVs. The detailed genomic characterization of HPV204, its tissue tropism, and potential clinical significance are currently under investigation and will be reported soon.

Nucleotide sequence accession number. The complete genome sequence of HPV2O4 is available in the ENA, GenBank, and DDBJ databases under the following accession number: KP769769.

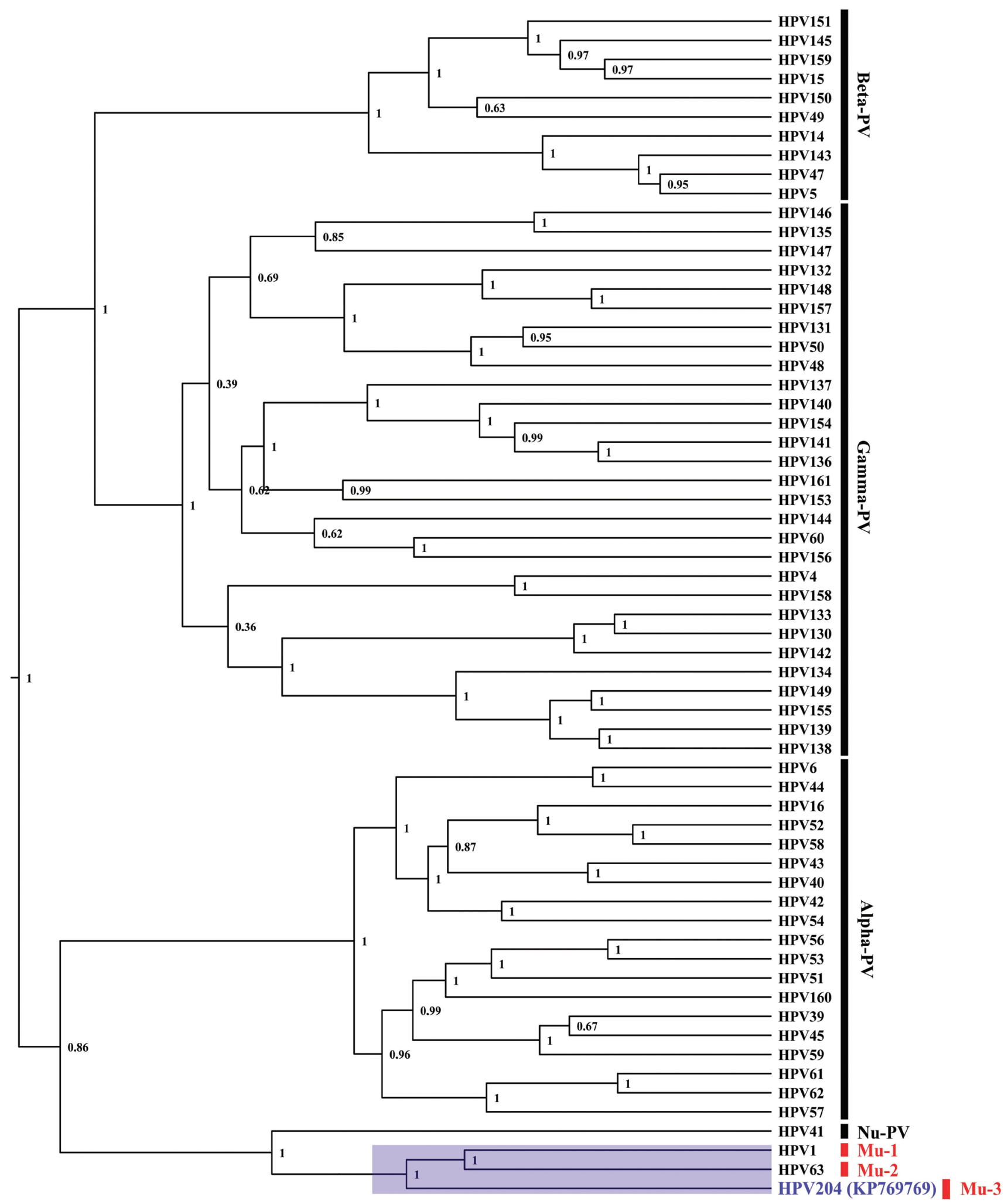

Figure 1 | Phylogenetic position of HPV204 (KP769769) in the Mu-PV genus. The numbers at the internal nodes represent Bayesian posterior probability values. 


\section{Acknowledgements}

This work is result of $\mathrm{PhD}$ research and is financially supported by the Slovenian Research Agency (ARRS), which funded AŠ through the Young Researcher Training Program, and in part by Slovenian-Argentinian Bilateral Project BI-AR-15-17-005 and by the European Commission's FP7 Program CoheaHr project (grant number: 603019).

\section{References}

1. Bernard HU, Burk RD, Chen Z, van Doorslaer K, zur Hausen H, de Villiers EM. Classification of papillomaviruses (PVs) based on 189 PV types and proposal of taxonomic amendments. Virology. 2010;401:70-9.

2. Cubie HA. Diseases associated with human papillomavirus infection. Virology. 2013;445:21-34.

3. de Villiers EM, Fauquet C, Broker TR, Bernard HU, zur Hausen H. Classification of papillomaviruses. Virology. 2004;324:17-27.

4. Kocjan BJ, Bzhalava D, Forslund O, Dillner J, Poljak M. Molecular methods for identification and characterization of novel papillomaviruses. Clin Microbiol Infect. 2015 May 20. [Epub ahead of print].

5. Van Doorslaer K, Tan Q, Xirasagar S, Bandaru S, Gopalan V, Mohamoud Y, et al. The Papillomavirus Episteme: a central resource for papillomavirus sequence data and analysis. Nucleic Acids Res. 2013;41:D571-8.

6. de Villiers EM. Cross-roads in the classification of papillomaviruses. Virology. 2013;445:2-10.

7. Danos O, Katinka M, Yaniv M. Molecular cloning, refined physical map and het erogeneity of methylation sites of papilloma virus type 1a DNA. Eur J Biochem. 1980;109:457-61.
8. Danos 0, Katinka M, Yaniv M. Human papillomavirus 1a complete DNA sequence: a novel type of genome organization among papovaviridae. EMBO J. 1982;1:231-6.

9. Egawa K, Delius H, Matsukura T, Kawashima M, de Villiers EM. Two novel types of human papillomavirus, HPV 63 and HPV 65: comparisons of their clinical and histological features and DNA sequences to other HPV types. Virology. 1993;194:789-99.

10. Bzhalava D, Eklund C, Dillner J. International standardization and classification of human papillomavirus types. Virology. 2015;476:341-4.

11. Chouhy D, Gorosito M, Sánchez A, Serra EC, Bergero A, Fernandez Bussy R, et al. New generic primer system targeting mucosal/genital and cutaneous human papillomaviruses leads to the characterization of HPV 115, a novel Beta-papillomavirus species 3. Virology. 2010;397:205-16.

12. Venuti A, Paolini F, Nasir L, Corteggio A, Roperto S, Campo MS, et al. Papillomavirus $E_{5}$ : the smallest oncoprotein with many functions. Mol Cancer. 2011;10:140. 